\title{
Lung sound localization using array of acoustic sensors
}

\author{
S.M. Akramus Salehin and Thushara D. Abhayapala \\ Applied Signal Processing Group \\ College of Engineering \& Computer Science \\ The Australian National University
}

\begin{abstract}
This paper presents a localization algorithm to detect lung sounds using an circular array of microphones. We use the natural basis functions of propagation waves in height invariant wavefields to form a spatial minimum variance (MV) problem in eigen space. We also derive a Nyquist criteria for localizing sources within a circular. Further, the Nyquist criteria shows that the radius of the region where sources can be localized is inversely proportional to the frequency of sound. The modified Nyquist criteria can be used for determining the number of sensors required for a required resolution, frequency range, and radius of the region for which sources need to be localized. The results are corroborated by computer simulations.
\end{abstract}

\section{INTRODUCTION}

Lung disorders or injury can result in changes in the spectral and/or spatial content of normal lung sounds. Localizing these lung sounds as an acoustic image can provide information to the extent and location of the disorder. Physicians have been using the stethoscope, since its invention in 1816, to detect and diagnose lung disorders. The use of a single stethoscope allows diagnosis from one location only at one instance and is qualitative being dependent on the skill of the physician. Multiple sensors to record data from several location simultaneously can be used to capture more information from the audible range of sounds produced by the lungs. However, localization of multiple nearfield sound sources within an array of sensors is a challenging signal processing problem, which has not been considered in the literature.

Localization of lung sounds from multi-sensor recordings can be classified as a problem of source localization within a circular array of sensors. Ward et. al. [1] used an optimization method to localize a single source in the interior of a sensor array. However, this optimization transformation cannot be applied when multiple sources are involved. In this paper, we propose an algorithm to localize multiple sources within a circular array of sensors using cylindrical harmonic functions of the wave equation. It is advantageous to use these functions since wave propagation through a layered cylindrical media can be analyzed by these functions and the chest can be modeled as cylindrical layered media.

Existing lung diagnosis methods involve ultrasound techniques. However, ultrasound techniques have poor performance with high frequency sounds [2]. Lung sound analysis with multiple stethoscopes can be used as a first diagnosis

S.M. Akramus has an association with National ICT Australia tool for the lung. Well established methods such as CT and Xrays can later be applied to confirm the results obtained form the multiple stethoscope device. Diseases or injury can cause alterations in the structure and function of the lungs, thus they can cause changes in lung sound production and transmission. Lung consolidation, pneumothorax and airway obstructions are a some of the conditions that can cause spectral and regional changes in lung sounds, if these changes are properly analyzed and localized from multi-sensor recordings then the extent and location of the trauma can be acquired [3], [4].

There are previous attempts to use multiple acoustic sensors in lung sound diagnostics [4]-[8] One of the earliest work on this was by Kompis et. al. who presented a solution for acoustic imaging of the human chest [4]. His algorithm was independent of the time of arrival of lung sounds and used a triangulation approach to locate sound sources from multiple sensor recordings. Others tried to either incorporate a more accurate acoustic transmission model of the lung at the expense of the localizing algorithm or proposed better localizing algorithms by simplifying the acoustic transmission model. Murphy assumed a isotropic velocity and calculated the locations of lung sounds based on differences in arrival times at the different sensors [7].

The aim of this paper is to propose and analyze sound localization algorithms within a circular sensor array for application to lung sound localization. However, the algorithms can be applied in sensor monitoring, hands free communication in rooms or for recording sounds in an auditorium. The solution to the Helmholtz wave equation can be synthesized and analyzed for a cylindrical co-ordinate system with a set of eigen basis functions. These eigen basis functions are the cylindrical harmonics. The lung can be modeled as a layered cylindrical structure and propagation in such environments can include reflection and refraction and can be analyzed using cylindrical harmonic functions [9]-[11]. This paper aims to develop localizing algorithms using these eigen basis functions and investigates their performance under different conditions. The localizing algorithms rely on the eigen basis decomposition since this will allow extension to a layered cylindrical model of lung sound propagation. Further, these eigen basis function were shown to be versatile for directionof-arrival (DOA) estimation [12].

The main contributions of the paper are described as follows:

- Propose and derive a spectral-based source localization 
algorithm using the natural basis functions of wave propagation in height invariant wavefields. Spectral based methods are more accurate than triangulation. Further, methods using differences in arrival times require higher precision equipment than spectral based algorithms.

- Performance of the proposed algorithm in terms of resolution, frequency range, number of sensors and radius of region for which sources can be localized is investigated.

\section{SYSTEM MODEL}

We assume a simple height invariant radius $R$ cylindrical chest model where the body is assumed to have a homogeneous medium. Consider $\mathrm{Q}$ sensors uniformly placed around the chest on a circle of radius of $R$. Let the sound sources are narrowband, zero mean, stationary point cylindrical sources with frequency $f$. This scenario is illustrated by Fig. 1 .

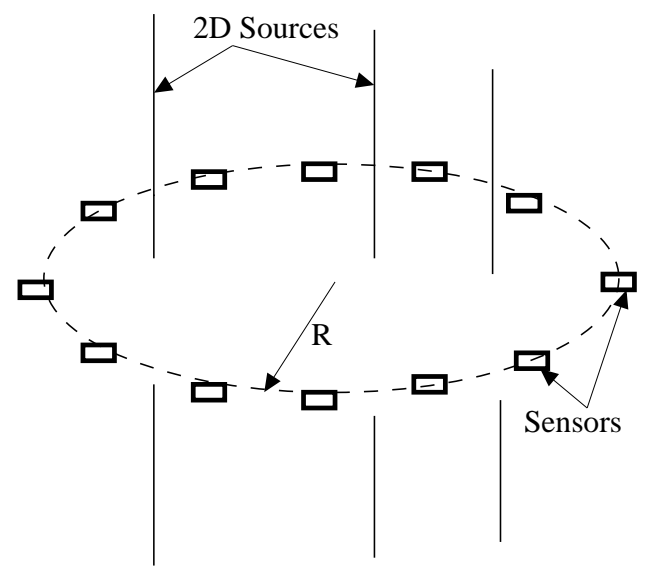

Fig. 1. The height invariant chest model.

Let $Q$ sensors be located $\boldsymbol{x}_{q} \equiv\left(R, \theta_{q}\right)$ with respect to a origin located at the center of the circle of radius $R$. We also assume that there are $V$ sources present within the region enclosed by the sensors. Let $\boldsymbol{y}_{v} \equiv\left(y_{v}, \phi_{v}\right)$ be the vector containing the location of the $v^{t h}$ source, where $y_{v}$ is the distance from the origin and $\phi_{v}$ is the angular position of the $v^{t h}$ source. We use wavenumber $k$ in this paper to represent frequency $f$ since $k=2 \pi f / c$ where $c$ is the speed of sound propagation. We can write the received signal at the $q$ th sensor as

$$
z_{q}(k)=\sum_{v=1}^{V} \frac{i}{4} H_{0}^{(2)}\left(k\left\|\boldsymbol{x}_{q}-\boldsymbol{y}_{v}\right\|\right) s_{v}(k)+n_{q}(k)
$$

where $H_{0}^{(2)}(\cdot)$ is the Hankel function of the second kind of order $0, s_{v}(k)$ is the signal form the $v$ th source, and $n_{q}(k)$ is the additive white Gaussian noise at the $q$ th sensor. Note that the factor $(i / 4) H_{0}^{(2)}\left(k\left\|\boldsymbol{x}_{q}-\boldsymbol{y}_{v}\right\|\right)$ is the fundamental solution to the wave equation in height invariant cylindrical coordinates $^{1}$. Now, we can write the received signal vector at the sensors as

$$
\boldsymbol{z}(k)=\sum_{v=1}^{V} \boldsymbol{a}\left(\boldsymbol{y}_{v}, k\right) s_{v}(k)+\boldsymbol{n}(k)
$$

\footnotetext{
${ }^{1}$ In 3D the fundamental solution $(1 / 4 \pi) \exp \left(j k\left\|\boldsymbol{x}_{q}-\boldsymbol{y}_{q}\right\|\right) /\left\|\boldsymbol{x}_{q}-\boldsymbol{y}_{q}\right\|$
}

where $\boldsymbol{z}(k)=\left[z_{1}(k), \ldots, z_{Q}(k)\right]^{T}, \boldsymbol{n}(k)$ is the $Q \times 1$ additive noise vector, and

$\boldsymbol{a}\left(\boldsymbol{y}_{v}, k\right)=\frac{i}{4}\left[H_{0}^{(2)}\left(k\left\|\boldsymbol{x}_{1}-\boldsymbol{y}_{v}\right\|\right), \cdots, H_{0}^{(2)}\left(k\left\|\boldsymbol{x}_{Q}-\boldsymbol{y}_{v}\right\|\right)\right]^{T}$

is termed as the array manifold vector.

In this paper, we only consider narrowband sources. This can be extended to the broadband case since broadband signals can be decomposed to a set of narrow band signals. By Rewriting (2) in matrix notation and ignoring $k$ for the narrowband case, we have

$$
z=\boldsymbol{A}(\mathbf{Y}) s+n
$$

where

$$
\boldsymbol{A}(\mathbf{Y})=\left[\boldsymbol{a}\left(\boldsymbol{y}_{1}\right), \ldots, \boldsymbol{a}\left(\boldsymbol{y}_{v}\right)\right]
$$

with unknown location vector $\mathbf{Y}=\left[\boldsymbol{y}_{1}, \ldots, \boldsymbol{y}_{v}\right]$, and $\boldsymbol{s}=$ $\left[s_{1}, \ldots, s_{v}\right]$.

The aim of the paper is, given the received sensor data $z$, to estimate the source locations $\mathbf{Y}$. We use the correlation matrix of the received data to develop a localization algorithm in this paper. Here we define the correlation matrix of the received sensor data as

$$
\mathbf{R}_{z}=E\left\{\boldsymbol{z} \boldsymbol{z}^{H}\right\}
$$

Substituting (4) into (6) and assuming that the noise and source signals are uncorrelated, we obtain the correlation matrix in terms of array manifold and source signals as

$$
\mathbf{R}_{z}=\boldsymbol{A}(\mathbf{Y}) E\left\{s \boldsymbol{s}^{H}\right\} \boldsymbol{A}(\mathbf{Y})+E\left\{\boldsymbol{n n} \boldsymbol{n}^{H}\right\}
$$

\section{EIGEN BASIS DECOMPOSITION}

The impinging wave field can be decomposed to a set of orthogonal basis functions dependent on the spatial coordinates used. These basis sets can be useful for synthesizing and analyzing wave field information captured by a sensor array. For a three dimensional wave field, spherical harmonics form the basis set and for a two dimensional wave field, as investigated in this paper, cylindrical harmonics form the basis set.

The two dimensional wave field investigated in this paper can be decomposed to basis functions by using the identity [13, page 66]

$$
H_{0}^{(2)}\left(k\left\|\boldsymbol{x}_{q}-\boldsymbol{y}_{v}\right\|\right)=\sum_{n=-\infty}^{\infty} H_{n}^{(2)}\left(k x_{q}\right) J_{n}\left(k y_{v}\right) e^{-i n \theta_{q}} e^{i n \phi_{v}}
$$

where $J_{n}(\cdot)$ is the Bessel function of order $n$. We substitute (8) in (1) to write

$$
\begin{aligned}
z_{q}(k)= & \sum_{n=-\infty}^{\infty}\left[\sum_{v=1}^{V} \frac{i}{4} J_{n}\left(k y_{v}\right) e^{i n \phi_{v}} s_{v}(k)\right] \\
& \times H_{n}^{(2)}\left(k x_{q}\right) e^{-i n \theta_{q}}+n_{q}(k) .
\end{aligned}
$$

The above decomposition consists of an infinite number of terms. The decomposition can be useful if the significant number of terms is finite.

The product of Hankel and Bessel functions approach zero as the order $n$ becomes large with respect to the argument. 
Therefore, for a finite region of space bounded by a circle of radius, $R$ and for the wave length of sound $\lambda$, the number of significant eigen basis functions can be limited without incurring significant errors [14] by truncating to

$$
M=\left\lceil\frac{\pi e R}{\lambda}\right\rceil
$$

terms. In the truncated 2D wave field decomposition, the order, $n$ spans the set $n \in[-M, \ldots, 0, \ldots, M]$ in (8).

\section{SOUND LOCALIZATION}

Using (8), we can separate the array manifold matrix $\boldsymbol{A}(\mathbf{Y})$ in to a product of two matrices:

$$
\boldsymbol{A}(\mathbf{Y})=\boldsymbol{\Gamma} \Upsilon
$$

where

$$
\boldsymbol{\Gamma}=\frac{i}{4}\left[\begin{array}{ccc}
H_{-M}^{(2)}(k R) e^{-i M \theta_{1}} & \ldots & H_{M}^{(2)}(k R) e^{i M \theta_{1}} \\
\vdots & \ddots & \vdots \\
H_{-M}^{(2)}(k R) e^{-i M \theta_{Q}} & \ldots & H_{M}^{(2)}(k R) e^{i M \theta_{Q}}
\end{array}\right]
$$

and

$$
\mathbf{\Upsilon}(\mathbf{Y})=\left[\begin{array}{ccc}
J_{-M}\left(k y_{1}\right) e^{i M \phi_{1}} & \ldots & J_{-M}\left(k y_{V}\right) e^{i M \phi_{V}} \\
\vdots & \ddots & \vdots \\
J_{M}\left(k y_{1}\right) e^{-i M \phi_{1}} & \ldots & J_{M}\left(k y_{V}\right) e^{-i M \phi_{V}}
\end{array}\right]
$$

Note that $\boldsymbol{\Gamma}$ contains only the location information of the

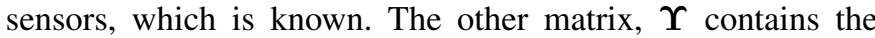
data on the source locations. Thus, we effectively separate the source locations from the sensor locations.

Now, we write the received array signal vector (4) as

$$
z=\Gamma \Upsilon(\mathbf{Y}) s+n .
$$

From the array vector, we can remove the contribution of sensor locations since the sensor locations are confined to the known matrix $\Gamma$. This can be done by multiplying the array data vector $z$ by the Moore-Penrose pseudo-inverse of $\boldsymbol{\Gamma}$. Thus,

$$
\begin{aligned}
\boldsymbol{\beta} & \triangleq \mathbf{G} \boldsymbol{z} \\
& =\mathbf{\Upsilon}(\mathbf{Y}) s+\widehat{\mathbf{n}}
\end{aligned}
$$

where $\mathbf{G}$ is the Moore-Penrose pseudo-inverse of $\boldsymbol{\Gamma}, \boldsymbol{\beta}$ is the transformed data, and $\widehat{\mathbf{n}}=\mathbf{G} \boldsymbol{n}$ is the modified noise. We can view this operation as a transformation of array data into cylindrical eigen domain, since this transformation effectively decomposed the received data into cylindrical modes.

One way of constructing $\mathbf{G}$ is

$$
\mathbf{G}=\left[\boldsymbol{\Gamma}^{T} \boldsymbol{\Gamma}\right]^{-1} \boldsymbol{\Gamma} .
$$

The calculation of the Moore-Penrose pseudo-inverse for $\boldsymbol{\Gamma}$ is equivalent to (17) only when $\Gamma$ is not close to being singular. A robust way to calculate $\mathbf{G}$ is to use the Singular Value Decomposition (SVD). The SVD of $\boldsymbol{\Gamma} \in \mathbb{C}^{Q \times(2 M+1)}$ is

$$
\boldsymbol{\Gamma}=\mathbf{U} \boldsymbol{\Xi} \mathbf{F}^{T}
$$

where $\mathbf{U} \in \mathbb{C}^{Q \times Q}$ and $\mathbf{F} \in \mathbb{C}^{(2 M+1) \times(2 M+1)}$ are orthogonal matrices, and $\boldsymbol{\Xi}$ is a $Q \times(2 M+1)$ diagonal matrix

$$
\boldsymbol{\Xi}=\left[\begin{array}{cccccc}
\xi_{1} & 0 & 0 & \ldots & 0 & 0 \\
0 & \xi_{2} & 0 & \ldots & 0 & 0 \\
0 & 0 & \xi_{3} & \ldots & 0 & 0 \\
\vdots & \vdots & \vdots & \ldots & \vdots & \vdots \\
0 & 0 & 0 & \ldots & \xi_{p} & 0
\end{array}\right]
$$

where $\xi_{i}$ are the singular values of $\boldsymbol{\Gamma}$ with $p=\min [Q, 2 M+$ 1] and $\xi_{1}>\xi_{2}>\ldots>\xi_{p}>0$.

From (19), $\mathbf{G}$ can be obtained by

$$
\mathbf{G}=\mathbf{U} \boldsymbol{\Xi}^{\dagger} \mathbf{F}^{T}
$$

where

$$
\boldsymbol{\Xi}^{\dagger}=\left[\begin{array}{cccccc}
\frac{1}{\xi_{1}} & 0 & 0 & \ldots & 0 & 0 \\
0 & \frac{1}{\xi_{2}} & 0 & \ldots & 0 & 0 \\
0 & 0 & \frac{1}{\xi_{3}} & \ldots & 0 & 0 \\
\vdots & \vdots & \vdots & \ldots & \vdots & \vdots \\
0 & 0 & 0 & \ldots & \frac{1}{\xi_{p}} & 0
\end{array}\right]
$$

For more information on the Moore-Penrose pseudo-inverse, the reader is referred to [15].

Now we can write the covariance matrix of transformed data $\beta$ as

$$
\hat{\mathbf{R}}_{\boldsymbol{\beta}} \triangleq E\left\{\boldsymbol{\beta}^{H} \boldsymbol{\beta}\right\} .
$$

In a practical scenario the covariance matrix $\hat{\mathbf{R}}_{\beta}$ is approximated by using number of (say $T$ ) snapshots as

$$
\hat{\mathbf{R}}_{\boldsymbol{\beta}} \approx \mathbf{R}_{\boldsymbol{\beta}} \triangleq \frac{1}{T} \sum_{t=1}^{T} \boldsymbol{\beta}(t)^{H} \boldsymbol{\beta}(t) .
$$

In the next step of the algorithm, we use Minimum Variance (MV) spatial spectrum estimation technique to obtain

$$
Z(y, \phi)=\frac{1}{\boldsymbol{c}(y, \phi)^{H} \mathbf{R}_{\boldsymbol{\beta}}^{-1} \boldsymbol{c}(y, \phi)}
$$

where

$$
\boldsymbol{c}(y, \phi)=\left[\begin{array}{c}
J_{-M}(k y) e^{i M \phi} \\
\vdots \\
J_{M}(k y) e^{-i M \phi}
\end{array}\right]
$$

The MV spatial spectrum $Z(y, \phi)$ can now be calculated and plotted over the whole range of $y$ and $\phi$ for all points interior to the sensor array. Then we estimate the source locations by locating the peaks in the MV spectrum.

\section{Theoretical Performance Analysis:Nyquist CRITERIA}

Note that the maximum angular frequency $n$ in (9) is $M=$ $\lceil\pi e \widetilde{R} / \lambda\rceil$ (given by (10)), if all the sources inside a region with radius $\widetilde{R}$. According to Nyquist's theorem, the sampling frequency must be greater than twice the highest frequency of the signal. Since we have $Q$ sensors over $2 \pi$ radians, we have

$Q>2 M$. 
Thus, to satisfy Nyquist's criteria, the following condition should be met

$$
\widetilde{R}<\frac{\lambda Q}{2 \pi e} .
$$

Works [16], [17] discussed spatial aliasing effects for the case of linear arrays. Spatial aliasing in linear arrays prevented localization of all sources. However, for localizing sources within a circular array, aliasing can be removed by reducing the radius of region, $\widetilde{R}$ where sources need to be located. As the frequency of sources increase, this radius reduces. This scenario contains an aliased region where $\widetilde{R}>\lambda Q /(2 \pi e)$ and a non-aliased region where $\widetilde{R}<\lambda Q /(2 \pi e)$.

The result from the Nyquist criteria gives an important interpretation towards sensor position in localizing sources. Assuming that we want to localize all sources within a radius, $\widetilde{R}$ then from the Nyquist criteria (27), the minimum number of sensors, $\widetilde{Q}$ required can be calculated. Further, these sensors can be placed at any radius greater that $\widetilde{R}$. Although, placing the sensors at a large radius can diminish their sensitivity to low power sources. In the sensor recording noise is present, therefore increasing the number of sensors from $\widetilde{Q}$ results in a better resolution since (23) become more accurate.

\section{Simulations}

A circular array consisting of 40 uniformly spaced sensors on the circumference of a circle is used to record sounds from the sources. The radius of this circle is set to 8 units. The average chest diameter varies according to gender. The average male and female chest diameter is approximately $30 \mathrm{~cm}$ and $26 \mathrm{~cm}$, respectively [18]. To correspond to a male chest, 1 unit needs to represent $1.875 \mathrm{~cm}$ and for a female chest, 1 unit needs to represent $1.625 \mathrm{~cm}$. We have used units since this allows the simulations to be scaled for a wide range of dimensions.

The source signals and the noise are modeled as stationary zero-mean white Gaussian processes. Further, the noise at each sensor is independent of the noise at any other sensor. The noise power received by the sensors is defined from the total signal power at the origin. For $V$ sources, the noise power, $\sigma_{N}^{2}$ received by the sensors is

$$
\sigma_{N}^{2}=\frac{\sum_{v=1}^{V} P_{v, 0}}{10^{\frac{S N R}{10}}}
$$

where $P_{v, 0}$ is the power of the $v^{t h}$ source at the origin.

The simulations are performed with narrowband sources and for each trial 100 snapshots are taken. The recorded signals are then discrete Fourier transformed within the desired frequency band.

We follow Section IV to obtain MV spatial spectral estimates. The MV spectral estimate shows peaks at locations where sound sources are present. This paper will not investigate the effect that increasing the number of sensors or the number of snapshots. These factors were previously investigated in works [19], [20] for linear arrays.

The environment consists of eight uncorrelated sound sources placed at different radii. Xs in Fig. 2a. shows the

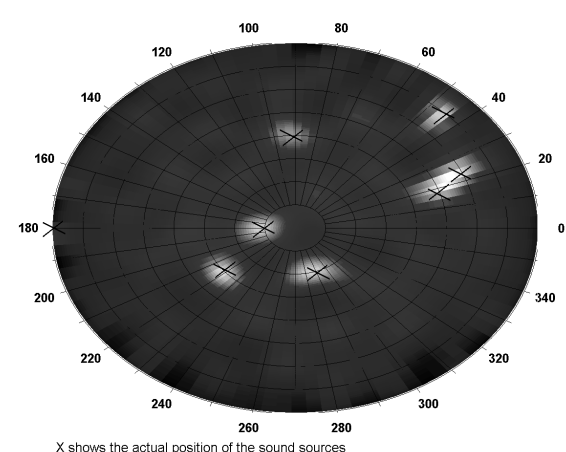

(a) The spatial spectrum as a polar plot with angle versus radius. Successive concentric circles represent an increase of one unit of distance from the center.

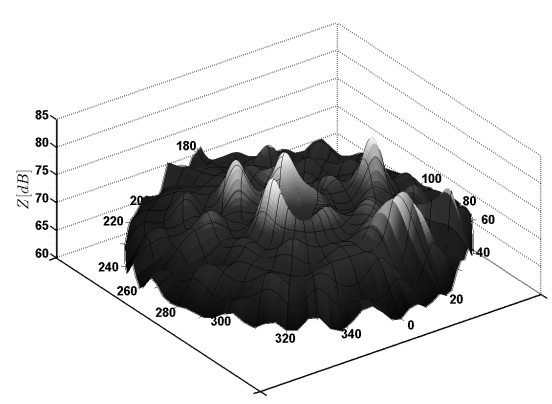

(b) 3-D plot of the spectrum.

Fig. 2. Spectrum for multiple 2-D sources with $\mathrm{SNR}=10 \mathrm{~dB}$.

actual locations of the eight sources. The SNR is set to 10 $\mathrm{dB}$ and the wavelength of the sources is 4 units. Scaling for an average male chest gives wavelength of the sound sources to be $7.5 \mathrm{~cm}$. The speed of sound in lung parenchyma varies between $25-75 \mathrm{~m} / \mathrm{s}$ [21]. Taking the lower speed, the frequency of the sound sources is $333 \mathrm{~Hz}$. Gavriely et al reported spectral characteristics of normal lung sounds to lie approximately between 50 and $1000 \mathrm{~Hz}$ [22], [23]. For lung sound localization, the performance of the algorithms are considered only within this frequency range.

The use of units for the radius and wavelength can be considered to be a powerful representation and allows the spectrum obtained to be flexible. The dimensions of the chest varies from one person to another. Suppose a lung sound localization device providing a spectral estimate for different people represents the radius in units which can be scaled for application to the specific chest diameter (measured beforehand with a tape measure). Further, the speed of sound in the lung varies. Therefore, wavelength represented in units can be scaled and represented to frequencies for different speeds using the relationship $c=f \lambda$.

The peaks in the MV spectral estimate in Fig. 2b. correspond accurately to actual source locations. In Fig. 2a., the peaks are represented by the light colored regions. Due to the definition of noise at the sensors (28), peaks decrease in height as the radius is increased. Further, the source lying on the same radius as the sensor radius (radius $=8$ units) cannot be detected. At SNR $10 \mathrm{~dB}$, the sources at radius of 5 and 
6 are detected as one source since the resolution is not high enough to give two peaks.

Further, the following comments pertaining to the proposed localizing algorithm can be made:

- The localizing algorithms can work without previous estimates of source locations.

- Since the algorithm calculates the covariance matrices for the modified sensor recordings, other spatial spectral methods such as MUSIC or its variants can be applied instead of MV spectrum.

- For large sensor arrays and considering the frequency of sound, the dimension can be reduced to $2 M+1$ by converting to the eigen basis sets of a $2 \mathrm{D}$ wave field. This reduces the computation expense of the proposed algorithms.

\section{CONCLUSIONS}

We have proposed an algorithm to localize sound sources within a circular array of sensors by decomposing the wave field into a set of eigen basis functions. The algorithm can be applied for acoustic imaging of the chest. However, in this paper we have assumed that the velocity of sound in the chest is isotropic. Future work will look at extending these algorithms for a layered cylindrical media that is characteristic of the chest and include reflections, refractions and standing waves. A Nyquist criteria is derived relating the number of sensors, frequency, and the radius of the region where the sources are located. We show that the Increase in frequency results in a reduction in the radius of the region for which sound sources are localized provided the number of sensors remain the same.

\section{REFERENCES}

[1] D. Ward and R. Williamson, "Beamforming for a source located in the interior of a sensor array," Signal Processing and Its Applications, 1999. ISSPA '99. Proceedings of the Fifth International Symposium on, vol. 2, pp. 873-876 vol.2, 1999.

[2] Z. Moussavi, Acoustic mapping and imaging of thoracic sounds. Morgan and Claypool Publishers, 2007, ch. 8, pp. 51-52.

[3] H. A. Mansy, S. J. Hoxie, W. H. Warren, R. A. Balk, R. H. Sandler, and H. A. Hassaballa, "Detection of Pneumothorax by Computerized Breath Sound Analysis," Chest, vol. 126, no. 4, pp. 881S-, 2004. [Online]. Available: http://meeting.chestjournal.org/cgi/content/abstract/126/4/881S

[4] M. Kompis, H. Pasterkamp, and G. R. Wodicka, "Acoustic Imaging of the Human Chest," Chest, vol. 120, no. 4, pp. 1309-1321, 2001. [Online]. Available: http://www.chestjournal.org/cgi/content/abstract/120/4/1309

[5] Charleston-Villalobos, S. and Cortés-Rubiano, S. and GonzálezCamerena, R. and Chi-Lem, G. and Aljama-Corrales, T., "Respiratory acoustic thoracic imaging (rathi): Assessing deterministic interpolation techniques," Med. Biol. Eng. Comput., vol. 42, no. 5, pp. 618-626, Sep 2004.

[6] S. Charleston-Villalobos, R. Gonzalez-Camarena, G. Chi-Lem, and T. Aljama-Corrales, "Acoustic thoracic images for transmitted glottal sounds," Engineering in Medicine and Biology Society, 2007. EMBS 2007. 29th Annual International Conference of the IEEE, pp. 34813484, Aug. 2007.

[7] J. Murphy, "Method and apparatus for locating the origin of intrathoracic sounds," U.S. patent, 729,272, Oct 1996.

[8] M. Ozer, S. Acikgoz, T. Royston, H. Mansy, and R. Sandler, "Boundary element model for simulating sound propagation and source localization within the lungs," J Acoust Soc Am., vol. 122, no. 1, pp. 657-661, Jul 2007.
[9] J. Barshinger and J. Rose, "Guided wave propagation in an elastic hollow cylinder coated with a viscoelastic material," Ultrasonics, Ferroelectrics and Frequency Control, IEEE Transactions on, vol. 51, no. 11, pp. 15471556, Nov. 2004

[10] C. Valle, J. Qu, and L. J. Jacobs, "Guided circumferential waves in layered cylinders," International Journal of Engineering Science, vol. 37, no. 11, pp. 1369-1387, Sep. 1999.

[11] G.-J. Yao, K.-X. Wang, J. Ma, and J. E. White, "Sh wavefields in cylindrical double-layered elastic media excited by a shear stress source applied to a borehole wall," Journal of Geophysics and Engineering, vol. 2, no. 2, pp. 169-175, 2005. [Online]. Available: http://stacks.iop.org/1742-2140/2/169

[12] T. Abhayapala and H. Bhatta, "Coherent broadband source localization by modal space processing," in 10th International Conference on Telecommunications (ICT 2003), vol. 2, February 2003, pp. 1617-1623.

[13] D. Colton and R. Kress, Inverse acoustic and electromagnetic scattering theory, 2nd ed. New York: Springer, 1998.

[14] R. Kennedy, T. Abhayapala, and H. Jones, "Bounds on the spatial richness of multipath," in 3rd Australian Communications Theory Workshop (AusCTW), February 2002, pp. 76-80.

[15] G. W. Stewart, "On the perturbation of pseudo-inverses, projections and linear least squares problems," SIAM Review, vol. 19, no. 4, pp. 634662, Oct 1977. [Online]. Available: http://www.jstor.org/stable/2030248

[16] D. Dudgeon, "Fundamentals of digital array processing," Proceedings of the IEEE, vol. 65, no. 6, pp. 898-904, June 1977.

[17] W. Kummer, "Basic array theory," Proceedings of the IEEE, vol. 80, no. 1, pp. 127-140, Jan 1992.

[18] G. Heinz, L. Peterson, R. Johnson, and C. Kerk, "Exploring relationships in body dimensions," Journal of Statistics Education, vol. 11, no. 2, 2003.

[19] Y. Bresler and A. Macovski, "On the number of signals resolvable by a uniform linear array," Acoustics, Speech, and Signal Processing [see also IEEE Transactions on Signal Processing], IEEE Transactions on, vol. 34, no. 6, pp. 1361-1375, Dec 1986.

[20] F. Li, H. Liu, and R. Vaccaro, "Performance analysis for doa estimation algorithms: unification, simplification, and observations," Aerospace and Electronic Systems, IEEE Transactions on, vol. 29, no. 4, pp. 1170-1184, Oct 1993.

[21] D. Rice, "Sound speed in pulmonary parenchyma," Journal of Applied Physiology, vol. 54, no. 1, pp. 304-308, 1983.

[22] N. Gavriely, Y. Palti, and G. Alroy, "Spectral characteristics of normal breath sounds," Journal of Applied Physiology, vol. 50, no. 2, pp. 307314, 1981.

[23] N. Gavriely, M. Nissan, A. Rubin, and D. Cugell, "Spectral characteristics of chest wall breath sounds in normal subjects," Thorax, vol. 50, no. 12, pp. 1292-1300, Dec 1995. 